\title{
Data reduction and calibration for LAMOST survey
}

\section{Ali Luo, Jiannan Zhang, Jianjun Chen, Yihan Song, Yue Wu, Zhongrui Bai, Fengfei Wang, Bing Du and Haotong Zhang}

National Astronomical Observatories, Chinese Academy of Sciences, Beijing China, email: lal@bao.ac.cn

\begin{abstract}
There are three data pipelines for LAMOST survey. The raw data is reduced to one dimension spectra by the data reduction pipeline(2D pipeline), the extracted spectra are classified and measured by the spectral analysis pipeline(1D pipeline), while stellar parameters are measured by LASP pipeline.

(a) The data reduction pipeline. The main tasks of the data reduction pipeline include bias calibration, flat field, spectra extraction, sky subtraction, wavelength calibration, exposure merging and wavelength band connection.

(b) The spectra analysis pipeline. This pipeline is designed to classify and identify objects from the extracted spectra and to measure their redshift (or radial velocity). The PCAZ ( Glazebrook et al. 1998) method is applied to do the classification and redshift measurement.

(c) Stellar parameters LASP. Stellar parameters pipeline (LASP) is to estimate stellar atmospheric parameters, e.g. effective temperature Teff, surface gravity $\log \mathrm{g}$, and metallicity $[\mathrm{Fe} / \mathrm{H}]$, for F, G and K type stars. To effectively determine those fundamental stellar measurements, three steps with different methods are employed. The first step utilizes the line indices to approximately define the effective temperature range of the analyzed star. Secondly, a set of the initial approximate values of the three parameters are given based on template fitting method. Finally,we exploit ULySS (Koleva et al. 2009) to give the final values of parameters through minimizing the $\chi^{2}$ value between the observed spectrum and a multidimensional grid of model spectra which is generated by an interpolating of ELODIE library. There are two other classification for A type star and M type star. For A type star, standard MK system is employed (Gray et al. 2009) to give each object temperature class and luminosity type. For M type star, they are classified into subclasses by an improved Hammer method, and metallicity of each objects is also given.

During the pilot survey, algorithms were improved and the pipelines were tested. The products of LAMOST survey will include extracted and calibrated spectra in FITS format, a catalog of FGK stars with stellar parameters, a catalog of M dwarf with subclass and metallicity, and a catalog of A type star with MK classification. A part of the pilot survey data, including about 319000 high quality spectra with SNR > 10, a catalog of stellar parameters of FGK stars and another catalog of a subclass of M type stars have been released to the public in August 2012 (Luo et al. 2012). The general survey started from October 2012, and completed the first year survey. The formal data release one (DR1) is being prepared, which will include both pilot survey and first year general survey, and planed to be released under the LAMOST data policy.
\end{abstract}

Keywords. methods: data analysis, techniques: spectroscopic, surveys, etc.

\section{References}

Glazebrook, K., Offer, A. R., \& Deeley, K. 1998, ApJ, 492, 98

Koleva M., Prugniel Ph., Bouchard A., \& Wu Y. 2009, A\&A, 501, 1269

Luo A., et al. 2012, Research in Aston. Astrophys, 12, 1243

Gray Richard O. \& Corbally, C. J. 2009, Stellar Spectral Classification. Princeton University Press, pp. 160-213. ISBN 0691125112. 